\title{
Artigo/Article
}

\section{Análise espacial da co-infecção tuberculose/HIV: relação com níveis socioeconômicos em município do sudeste do Brasil}

\author{
Spatial analysis of tuberculosis/HIV coinfection: its relation with socioeconomic levels in a \\ city in south-eastern Brazil
}

\begin{abstract}
Silvia Helena Figueiredo Vendramini ${ }^{1}$, Natália Sperli Geraldes Marin dos Santos ${ }^{2}$, Maria de Lourdes Sperli Geraldes Santos ${ }^{1}$, Francisco Chiaravalloti-Neto ${ }^{3}$, Maria Amélia Zanon Ponce ${ }^{4}$, Claudia Eli Gazetta ${ }^{1}$, Tereza Cristina Scatena Villa ${ }^{5}$ Antonio Ruffino Netto ${ }^{6}$
\end{abstract}

\section{RESUMO}

Introdução: Analisar espacialmente a co-infecção tuberculose/vírus da imunodeficiência humana e associá-la com variáveis socioeconômicos, São José do Rio Preto, SP, 1998-2006. Métodos: Foram geocodificados casos novos de TB/HIV e calculados coeficientes de incidência segundo unidades espaciais. Utilizou-se o índice de Moran para avaliar a dependência espacial das incidências. Regressões múltiplas foram realizadas para selecionar variáveis com maior poder de explicação da dependência espacial. O indicador local de associação espacial foi utilizado para identificação de agrupamentos espaciais significantes. Resultados: $O$ índice de Moran foi de 0,0635 ( $p=0,0000)$, indicando ocorrência de dependência espacial. A variável que apresentou maior poder de explicação da dependência espacial da incidência foi a porcentagem de chefes de família com até três anos de instrução. O LISA cluster map para os coeficientes de incidência de co-infecção TB/HIV evidenciou aglomerados de alta incidência na região norte e baixa incidência na sul e oeste do município. Conclusões: $\mathrm{O}$ estudo possibilitou a compreensão da distribuição geográfica espacial da co-infecção TB/HIV no município e apontou a sua associação com variáveis socioeconômicas dando subsídios para o planejamento orientado para a priorização das regiões com maior carência social e consequentemente maiores incidências da doença.

Palavras-chaves: Tuberculose. HIV. Distribuição espacial. Condição social.

\begin{abstract}
Introduction: Spatial analysis of the distribution of tuberculosis/HIV coinfection was performed and associated with socioeconomic indicators in São José do Rio Preto, from 1998 to 2006. Methods: New TB/HIV coinfection cases were georeferenced and incidence coefficients were calculated for spatial units. Moran's index was used to evaluate spatial associations of incidences. Multiple regressions selected variables that could best explain the spatial association of incidences. The local indicator of spatial association was used to identify significant spatial groupings. Results: Moran's index was 0.0635 ( $p=0.0000)$ indicating that the incidence association occurred. The variable that best explained the spatial association of incidence was the percentage of heads of families with up to three years of education. The LISA cluster map for TB/HIV coinfection incidence coefficients showed groups with high incidence rates in the North and low incidence in the South and West regions of the municipality. Conclusions: The study elucidated the spatial geographic distribution of TB/HIV coinfection and determined its association with socioeconomic variables, thus providing data for oriented planning, prioritizing socially disadvantaged regions that present a higher incidence of the disease.
\end{abstract}

Key-words: Tuberculosis. HIV. Spatial distribution. Social conditions.

1. Departamento de Enfermagem em Saúde Coletiva e Orientação Profissional, Faculdade de Medicina de São José do Rio Preto, São José do Rio Preto, SP. 2. Programa de Pós-Graduação em Ciências da Saúde, Faculdade de Medicina de São José do Rio Preto, São José do Rio Preto, SP. 3. Departamento de Epidemiologia, Faculdade de Saúde Pública da Universidade de São Paulo, São Paulo, SP. 4. Programa de Pós-Graduação em Enfermagem em Saúde Pública, Escola de Enfermagem de Ribeirão Preto, Universidade de São Paulo, Ribeirão Preto, SP. 5. Escola de Enfermagem de Ribeirão Preto, Universidade de São Paulo, Ribeirão Preto, SP. 6. Departamento de Medicina Social, Faculdade de Medicina de Ribeirão Preto, Universidade de São Paulo, Ribeirão Preto, SP.

Endereço para correspondência: Profł ${ }^{\mathrm{S}}$ Silvia Helena Figueiredo Vendramini. FAMERP/DESCOP. Av Brigadeiro Faria Lima 5416, Vila São Pedro, 15090-000 São José do Rio Preto, SP.

Tel: 5517 3201-5721

e-mail: silviahve@gmail.com

Recebido para publicação em 08/04/2010

Aceito em 13/07/2010

\section{INTRODUÇÃO}

O vírus da imunodeficiência humana (HIV) tem contribuído para o incremento da epidemia da tuberculose (TB), dificultando o seu controle no mundo, principalmente em populações de baixa renda, nas quais sua associação com o HIV representa a principal causa de morte ${ }^{1}$.

As duas doenças (principalmente a TB) estão concentradas em áreas de pobreza, onde existem recursos mínimos para o diagnóstico, tratamento e controle da infecção e os serviços de saúde pública não atendem às necessidades para o controle das epidemias, apesar das grandes conquistas no tratamento tanto da TB como da $\operatorname{AIDS}^{2,3}$.

Quanto ao aspecto biológico, a combinação das duas doenças é preocupante, pois uma promove o agravamento da outra, acelerando a dupla epidemia ${ }^{4}$. Este impacto negativo de uma doença sobre a outra dificulta o alcance das metas estabelecidas para o controle da TB no Brasil, havendo a necessidade de implementação de ações de controle da coinfecção TB/HIV nas áreas assistencial, de vigilância epidemiológica e programática ${ }^{5}$.

Também, existem as flagrantes desigualdades econômicas, sociais, regionais, étnicas e de gênero, além da fragilidade da comunicação entre o Programa Nacional de Controle da Tuberculose (PNCT) e o Programa Nacional de Doenças Sexualmente Transmissíveis e AIDS (PN-DST/AIDS) que influenciam nos aspectos epidemiológicos da coinfecção TB/HIV ${ }^{6-9}$.

É importante ressaltar que no Brasil, em 2006, $8 \%$ dos casos de TB ocorreram em indivíduos com sorologia positiva para HIV, no Estado de São Paulo e São José do Rio Preto, as taxas foram respectivamente de $14 \%$ e $26 \%{ }^{10}$.

A alta taxa de co-infecção TB/HIV encontrada no município, quando comparada ao país e ao estado, merece maior atenção e requer a incorporação de métodos da epidemiologia, da geografia e da estatística que permitam uma análise integrada da associação 
deste evento com indicadores e/ou variáveis socioambientais que auxiliem as políticas públicas de saúde na incorporação de ações inovadoras no controle da dupla epidemia ${ }^{11-19}$.

Neste contexto, ressalta-se a importância de construir indicadores sociais para desvendar as desigualdades socioespaciais como fatores fortemente marcados pela exclusão social, com impacto sobre o processo saúde doença das populações. Dentro desta perspectiva é possível analisar e caracterizar espacialmente as áreas de risco para co-infecção TB/HIV.

O estudo tem o objetivo de analisar espacialmente a co-infecção TB/HIV em município de grande porte da região sudeste do Brasil e associá-la a indicadores socioeconômicos no período de 1998 a 2006.

\section{MÉTODOS}

\section{Área de estudo}

Município de São José do Rio Preto, com população estimada em 2006 de 415.508 habitantes, está localizado ao norte do estado de São Paulo, Brasil (2049'11' S e 49²2'46’ W), com área total de $434,10 \mathrm{~km}^{2}$ e área urbana de $96,81 \mathrm{~km}^{2}$.

\section{População de estudo}

Foram selecionados para o estudo, os casos novos de co-infecção TB/HIV residentes no município, identificados no período de janeiro de 1998 a dezembro de 2006 por ocasião do diagnóstico de TB e realização de sorologia anti-HIV.

\section{Georreferenciamento dos casos}

O processo de geocodificação foi obtido pela equiparação dos endereços dos casos de TB/HIV com mapa de segmento de logradouros fornecido pela Prefeitura Municipal, usando o software ArcGIS 9.1. A geocodificação foi obtida pela interpolação linear do número do endereço a um ponto no segmento de logradouro correspondente, situado entre dois pontos que definem a faixa de numeração daquele segmento de logradouro.

\section{Unidades de agregação}

Foram utilizadas, como unidades de agregação, 108 unidades espaciais criadas a partir do agrupamento de setores censitários em área urbana do município. Cada unidade espacial resultou do agrupamento de quatro setores censitários do IBGE (Fundação Instituto de Geografia e Estatística), procurando-se respeitar delimitadores geográficos, como rios e rodovias, assim como manter similaridade na forma geométrica de cada unidade.

\section{Análise dos dados}

Os casos geocodificados de TB/HIV foram agrupados segundo as unidades espaciais e calcularam-se, para cada unidade, coeficientes de incidência de TB/HIV, pela divisão entre o número de casos de TB/HIV de cada unidade e sua respectiva população, multiplicada por 100.000. Trabalhou-se com a raiz quadrada do coeficiente de incidência (variável dependente), por esta ter uma melhor aproximação para a distribuição normal.

Inicialmente, foi verificada a existência de autocorrelação espacial entre as raízes dos coeficientes de incidência de TB/HIV. Uma vez que esta foi identificada, utilizou a metodologia proposta por Cliff \& $\mathrm{Ord}^{20}$, apropriada para dados de área, adequada para estudar a distribuição espacial de algum fenômeno e levar em conta de dependência espacial dos dados ou os efeitos de segunda ordem ${ }^{21}$.
Para cumprir o objetivo proposto, isto é, verificar quais variáveis socioeconômicas e demográficas teriam maior poder explicativo sobre a incidência de TB/HIV, foi feita a suposição de que todas as informações analisadas apresentavam homogeneidade dentro das unidades de agregação utilizadas neste estudo. Isto parece razoável, uma vez que os setores censitários são unidades homogêneas utilizadas pelo IBGE para realização de censos demográficos, consideração que pode ser estendida para as unidades espaciais, uma vez que resultaram do agrupamento de quatro setores.

As variáveis socioeconômicas e demográficas (independentes) foram obtidas a partir de informações disponibilidades pelo IBGE para o censo demográfico de 2000, sendo escolhidas aquelas variáveis ou as correspondentes transformações pela raiz quadrada que apresentaram uma boa aproximação para a distribuição normal. A avaliação da normalidade foi realizada pela aplicação do teste Shapiro-Wilk ${ }^{22}$.

Para mensurar a autocorrelação espacial dos coeficientes de incidência de TB/HIV, utilizou-se o índice de Moran, dado por:

$$
I=\frac{n \sum_{i} \sum_{j} w_{i j} z_{i} z_{j}}{S_{0} \sum_{i} z_{i}^{2}}
$$

onde:

$i, j=1, \ldots, 108$ unidades espaciais

$$
\mathrm{S}_{0}=\sum_{i} \sum_{j} W_{i j}
$$$$
\mathrm{Z}_{i}=x_{i}-\bar{x}
$$

$x_{i}=$ raiz quadrada do coeficiente de incidência de TB/HIV

$w_{i j}=$ ponderação espacial atribuída ao par de unidades espaciais.

O índice de Moran foi calculado com ferramentas do software ArcGIS 9.3 em que as ponderações $w_{i j}$ foram o inverso da distância entre os centróides das áreas $i$ e $j$. A matriz de vizinhança para as 108 unidades espaciais foi definida pelas unidades contíguas, com pelo menos um ponto em comum. O Índice de Moran é entendido como a correlação entre valores de uma variável avaliada, neste caso, em setores vizinhos $(i \mathrm{e} j)$. A variação deste índice está entre -1 e +1 . Para testar este coeficiente de autocorrelação, existe uma hipótese nula de não correlação espacial e outra, em que há expressão da proximidade entre áreas geográficas, definida pela matriz de ponderações $w_{i j}$, $\mathrm{e}$ ponderações sobre critérios de vizinhança ${ }^{22}$.

O teste de significância I foi feito para testar a hipótese da independência espacial, levando-se em consideração a normalidade das observações. O índice padronizado foi calculado, de acordo com a seguinte fórmula:

$$
I_{p a d r}=\frac{I-E(I)}{\sqrt{\operatorname{Var}}(I)}
$$

onde: $\mathrm{E}(\mathrm{I})$ = média do índice de Moran e Var (I) = variância do índice de Moran.

No caso de significância estatística do índice padronizado para a variável raiz quadrada do coeficiente de incidência de TB/HIV, regressões múltiplas foram feitas utilizando-se o programa STATA 
para a seleção das variáveis socioeconômicas e demográficas que poderiam ser os fatores mais explicativos das incidências nas diversas unidades espaciais consideradas. Em cada passo, foram calculados os resíduos das variáveis independentes e, a partir deles, calcularam-se novos índices de Moran e seus índices padronizados, até sua minimização e diminuição de significância. Por meio deste procedimento, as variáveis mais explicativas são apontadas e, a partir do controle dos seus efeitos nos resíduos do modelo, a autocorrelação espacial perde a significância estatística que possuía no início.

O procedimento utilizado foi o passo a passo proposto por Anselin $^{23}$, para minimizar o coeficiente $I_{\text {padr }}$, calculado por meio dos resíduos da regressão da variável resposta com as variáveis independentes, até que este perca sua significância.

O indicador local de autocorrelação espacial (LISA) ${ }^{23}$ foi adotado como índice para identificar agrupamentos espaciais de coeficientes de incidência de TB/HIV significantes. A definição do índice LISA é dada por:

$$
I(i)=\left(\frac{X i-\bar{X}}{\delta}\right) \cdot \sum_{j=1}^{n}\left[W i j \cdot \frac{(X j-\bar{X})}{\delta}\right]
$$

onde $I(i)$ é o LISA da região $i, W i j$ é a proximidade da região $i$ à região $j, X i$ é o valor do coeficiente de incidência de TB/HIV do setor censitário $i, X j$ é o valor do coeficiente de incidência de TB/HIV da unidade espacial $j, \bar{X}$ é o valor médio do coeficiente de incidência, $\delta$ é o desvio padrão de $X i$ e $n$ é o número total de regiões avaliadas. O termo $W i j$ descreve a proximidade da região $i$ à região. Se a região $i$ é adjacente à região $j$, o valor 1 é atribuído; caso contrário, o valor 0 é atribuído.

Esta análise, que foi realizada através do Programa GeoDa 0.9.5-i, forneceu o LISA cluster map, onde são apresentados as unidades espaciais que apresentaram valores significantes do LISA classificadas em quatro possibilidades: high-high e low-low, representando unidades espaciais com altas e baixas incidências circundadas por unidades também com altas e baixas incidências, respectivamente; high-low e low-high, representando unidades com baixas e altas incidências circundadas por unidades com altas e baixas incidências, respectivamente. As duas primeiras categorias representam áreas de concordância e as duas últimas áreas de transição.

\section{RESULTADOS}

Dos 307 casos de TB/HIV notificados na área urbana do município, foram geocodificados 262 e excluíram-se 45 (14,5\%): 12 em situação prisional ou com duplicidade de endereço e 33 por inexistência/inadequação de endereços com a base cartográfica.

Na Tabela 1, são apresentados os resultados da análise estatística espacial da variável resposta, a raiz quadrada do coeficiente de incidência de TB/HIV, entre janeiro de 1998 e dezembro de 2006. No período estudado, o índice de Moran foi de 0, 0635 ( $\mathrm{p}=$ 0, 0000) o que indica ocorrência de dependência espacial ou efeitos de segunda ordem. As variáveis independentes estão dispostas na Tabela 1 por ordem crescente de valores de I e decrescente de valores de $\mathrm{p}$.

Logo no primeiro passo, seis variáveis (porcentagem de chefes de familia com até três anos de instrução, proporção de mulheres analfabetas, porcentagem de pessoas com renda de 2 a 3 salários mínimos, porcentagem de chefes de familia analfabetos, proporção de pessoas analfabetas e porcentagem de chefes com 11 a 14 anos de instrução) revelaram associação com a incidência de TB/HIV e eliminaram a dependência espacial apontada
TABELA 1 - Índice de Moran (I) para a raiz do coeficiente de incidência de TB/HIV e variáveis socioeconômicas e demográficas, São José do Rio Preto, 1998 a 2006.

\begin{tabular}{lcc}
\hline & \multicolumn{2}{c}{ Índice de Moran } \\
\cline { 2 - 3 } Variáveis & Valor de I & Valor de p \\
Raiz da incidência & 0,0635 & 0,0000 \\
\hline Ajustada por: & & \\
Porcentagens de chefes com até três anos de instrução & $-0,0096$ & 0,5106 \\
Proporção de mulheres analfabetas & $-0,0047$ & 0,3323 \\
Porcentagem de pessoas com renda de 2 a 3 salários mínimos & $-0,0023$ & 0,2547 \\
Raiz da porcentagem de chefes de família analfabetos & $-0,0022$ & 0,2519 \\
Proporção de pessoas analfabetas & $-0,0019$ & 0,2428 \\
Porcentagem de chefes com 11 a 14 anos de instrução & 0,0027 & 0,1288 \\
Porcentagem de chefes de família com 8 a 10 anos de instrução & 0,0173 & 0,0062 \\
Raiz da proporção de domicílios com 5 ou mais moradores & 0,0200 & 0,0029 \\
Número de moradores por domicílio & 0,0288 & 0,0002 \\
Raiz da porcentagem de mulheres chefes de família & 0,0570 & 0,0000 \\
Raiz do número de moradores por área $\left(\mathrm{km}^{2}\right)$ & 0,0583 & 0,0000 \\
Raiz do número de domicílios por área $\left(\mathrm{km}^{2}\right)$ & 0,0633 & 0,0000 \\
\hline
\end{tabular}

inicialmente, isto é, os resíduos da análise de regressão mostraram-se independentes, condição necessária para a validade da técnica estatística utilizada. Destas, a que apresentou a maior diminuição do índice de Moran foi a porcentagem de chefes de familia com até três anos de instrução. $\mathrm{O}$ índice de Moran, calculado para o resíduo desta variável foi o menor e o menos significativo ( $I=-0,0096 ; \mathrm{p}=0,5106)$. As outras variáveis citadas também produziram índices de Moran não significantes, mas com maiores valores do índice e menores valores de $\mathrm{p}$.

A Figura 1 apresenta os coeficientes de incidência por TB/HIV divididos em tercis para as unidades espaciais urbanas do município.

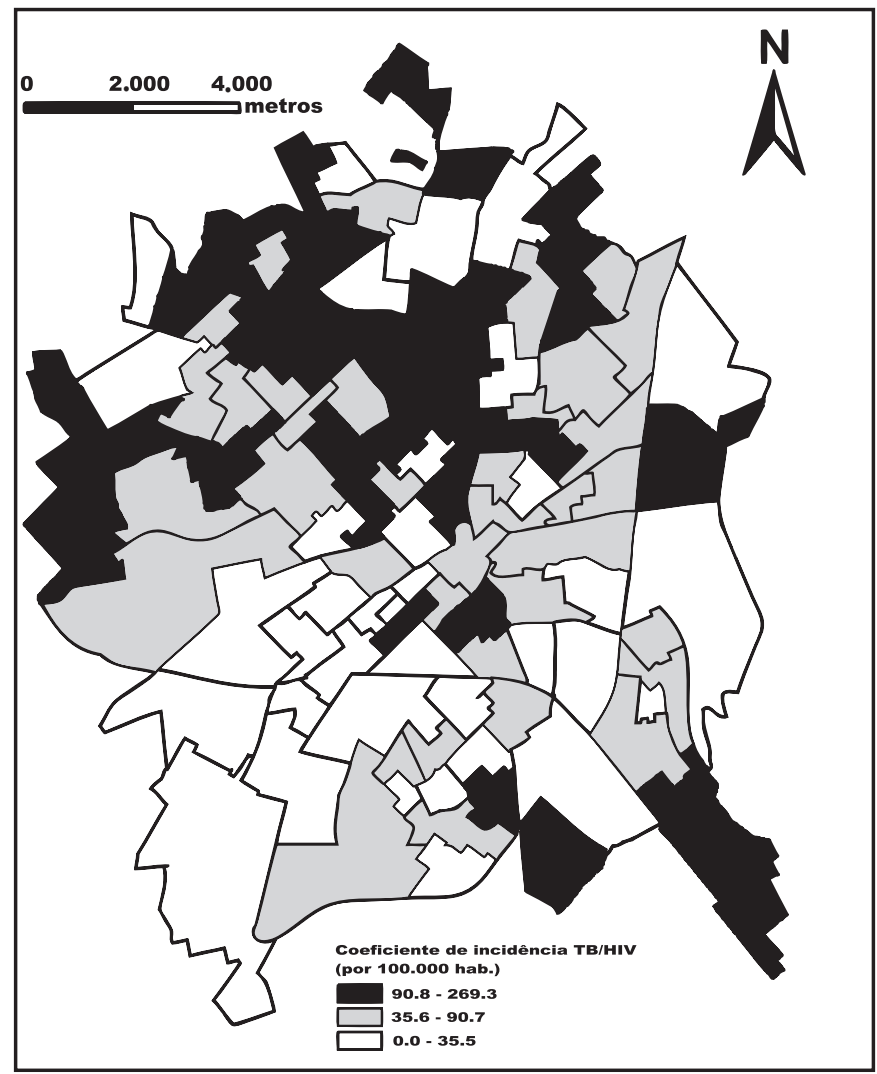

FIGURA 1 - Coeficientes de incidência de TB/HIV das unidades espaciais da área urbana de São José do Rio Preto, 1998 a 2006. 
A maioria das unidades com incidências acima do segundo tercil concentrou-se nas áreas norte e sudeste e a maioria das unidades com valores abaixo do primeiro tercil localizou-se nas áreas sudoeste e leste. A Figura 2 apresenta as porcentagens dos chefes de família com até três anos de instrução por tercis. A Figura 3 apresenta o mapa temático com os resíduos da regressão entre as raízes das incidências de TB/HIV e a variável independente porcentagem de chefes de familia com até três anos de instrução, para os quais a análise pelo índice de Moran mostrou a inexistência de efeitos de segunda ordem.

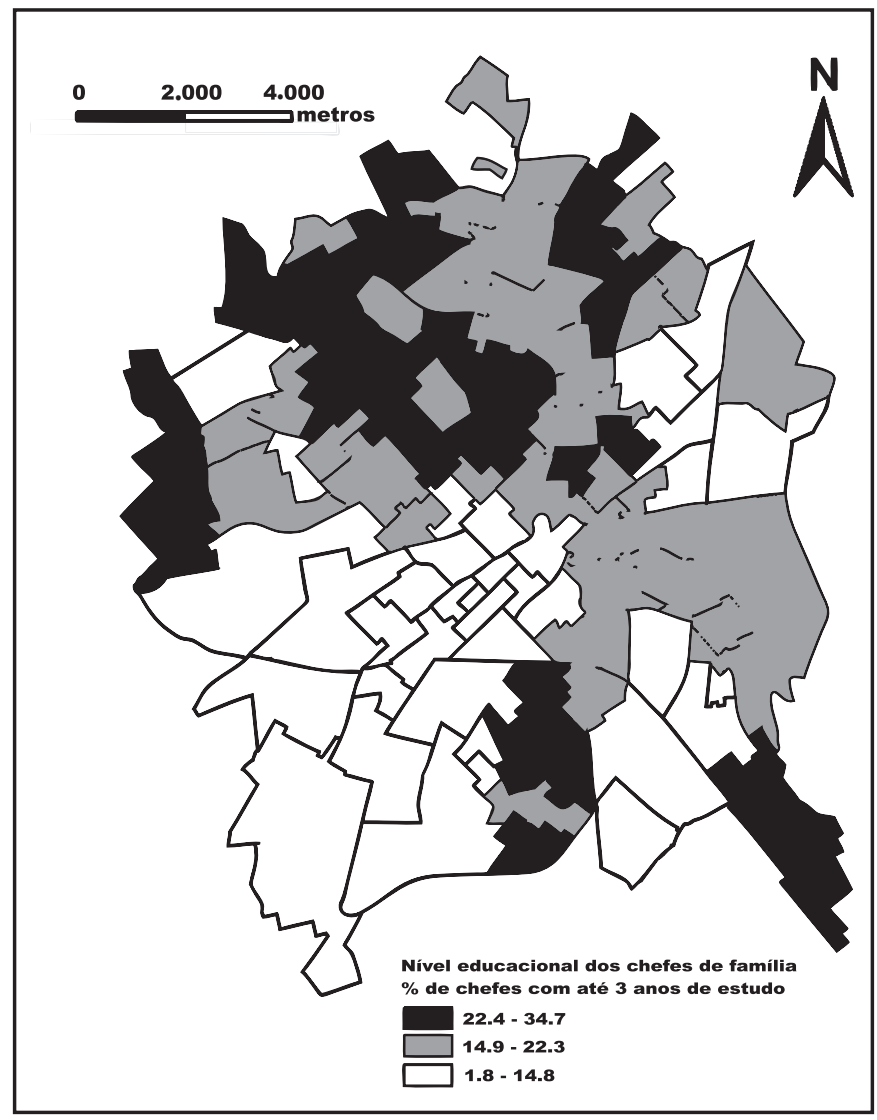

FIGURA 2 - Nível de escolaridade de chefes de família das unidades espaciais (procentagem de chefes de família com até 3 anos de instrução) da área urbana de São José do Rio Preto, 2000.

A comparação das Figuras 1 e 2 mostra a existência de associação entre a incidência de TB/HIV e a proporção de chefes com até três anos de instrução. Muitas das unidades espaciais classificadas acima do segundo tercil de incidência coincidem com aquelas que apresentaram as maiores proporções de chefes de família com até 3 anos de instrução. A análise conjunta das Figuras 1 e 2 aponta para algumas discordâncias. No centro da área sul, ocorreram unidades espaciais com baixa incidência, mas classificadas acima do segundo tercil da proporção de chefes com baixa instrução. O mesmo ocorreu em duas unidades no extremo norte e em duas no centro da área urbana. Em uma unidade localizada no oeste e outra no leste da área urbana, ocorreu o inverso; unidades com altas incidências e baixas proporções de chefes com pouca instrução.

A Figura 4 apresenta o LISA cluster map para os coeficientes de incidência de TB/HIV. As unidades classificadas como high-high, localizadas na área norte da cidade, formaram os aglomerados de alta incidência, circundados por outros com valores também altos. Os aglomerados de unidades com baixa incidência (low-low) estavam localizados, predominantemente, na área sudoeste da cidade.

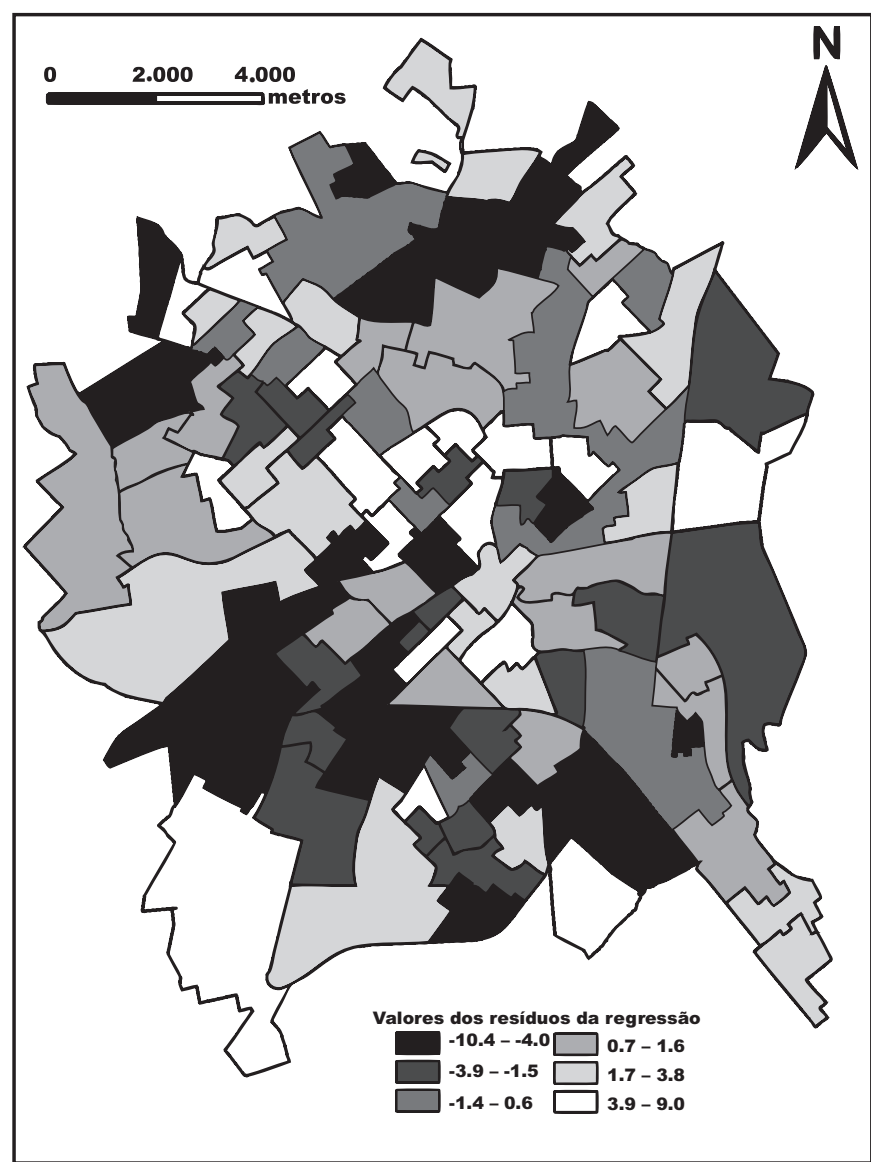

FIGURA 3 - Resíduos da regressão entre a raiz quadrada das incidência de TB/HIV e a porcentagem de chefes de família com até três anos de escolaridade ( de Moran =-0,0096; $p=0,5106$ ), área urbana de São José do Rio Preto.

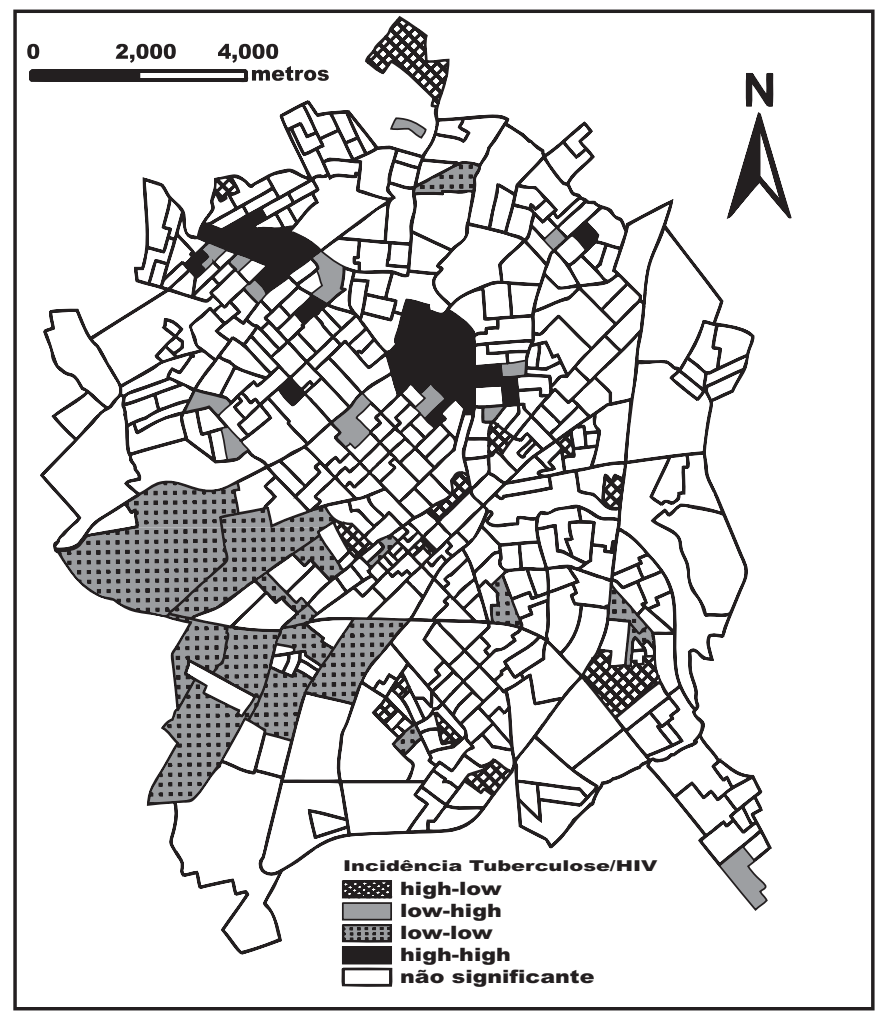

FIGURA 4 - LISA cluster map para os coeficientes de incidência de TB/HIV: setores censitários com LISA estatisticamente significante classificados por tipo de correlação espacial, São José do Rio Preto, 1998 a 2006. 


\section{DISCUSSÃo}

O município em estudo apresentou, em 2006, um coeficiente de incidência de TB de 34/100.000 habitantes, menor que os valores para o Estado de São Paulo (ESP) (44/100.000 habitantes) e o Brasil (42/100.000 habitantes). Em relação à AIDS, a situação é inversa: 32/100.000 habitantes, 21/100.000 habitantes e 17/100.000 habitantes, respectivamente. No mesmo ano, em São José do Rio Preto, no ESP e no Brasil, a incidência da coinfecção TB/HIV foi de 9/100.000 habitantes, $6 / 100.000$ habitantes e $3 / 100.000$ habitantes, respectivamente, acompanhando o comportamento epidemiológico da $\operatorname{AIDS}^{10}$. Esta situação evidencia o impacto da AIDS na TB e desta na AIDS, considerando que o HIV potencializa a epidemia da TB, assim como esta é a principal causa de morte nas pessoas vivendo com HIV/AIDS (PVHS) ${ }^{3}$.

A TB e a AIDS são doenças de magnitude tal, que ultrapassam as barreiras biológicas, constituindo em grave problema social. A vulnerabilidade dos indivíduos se dá em não se perceberem em risco, diminuindo a prática do autocuidado, além de terem maior dificuldade de acesso aos serviços de saúde ${ }^{24}$. Além disso, a predominância da co-infecção em indivíduos com baixa escolaridade reflete a situação educacional do Brasil, caracterizada pelo analfabetismo funcional (a alfabetização somente se consolida entre as pessoas que completaram a $4^{\mathrm{a}}$ série do ensino fundamental), sendo que, $27,8 \%$ da população de 15 anos ou mais de idade se encontram nessa situação em consequência da evasão escolar, observada no sistema de ensino ${ }^{25-26}$.

Os resultados deste estudo apontaram para a existência de efeitos de primeira e segunda ordem (dependência espacial) nos coeficientes de incidência da co-infecção TB/HIV. A análise de regressão desta variável com várias variáveis socioeconômicas (Tabela $\mathbf{1}$ ), uma a uma, mostrou que ambos os efeitos foram controlados, uma vez que os resíduos das várias regressões apresentaram índice de Moran não significantes, condição para que as regressões possam ser utilizadas, isto é, independência dos resíduos. As variáveis socioeconômicas associadas, neste estudo, com as incidências são consideradas bons indicadores da estratificação social, que, por sua vez, é considerada preditiva das condições de saúde das populações. Os resultados deste estudo revelaram que o padrão de risco é pior para os indivíduos pertencentes aos grupos sociais menos privilegiados ${ }^{27}$.

A porcentagem de chefes de família com até três anos de instrução como variável mais explicativa da incidência de TB/HIV coincide com a consideração do nível educacional como bom indicador socioeconômico. A educação é um importante determinante do nível de saúde, é de fácil obtenção, é impermeável às perdas impostas por doenças associadas ao preconceito e ao estigma, como a TB e a AIDS, e mantémse praticamente inalterado por todo o curso da vida adulta ${ }^{22,27,28}$.

São José do Rio Preto está classificado como um dos melhores municípios em qualidade de vida, no Estado de São Paulo e Brasil ${ }^{29}$. Quando considerado como um todo, apresenta boa situação socioeconômica e tem padrões urbanos e de condições de vida melhores que muitas cidades do mesmo porte no país e na América Latina ${ }^{6,7}$.

Porém, quando o município é analisado segundo unidades espaciais, observam-se as aglomerações populacionais nas periferias, caracterizadas por piores condições sociais individuais (grau de instrução) e coletivas (privação social e marginalidade).

Desta maneira, a distribuição da população no espaço urbano segue os mesmos padrões observados nos grandes centros ${ }^{6}$, caracterizados por um espaço intraurbano desigual, segmentado e excludente. Os resultados revelam uma nítida separação entre riqueza e a pobreza, havendo uma predominância nas regiões centro-sudoeste e leste para os setores de inclusão social e uma maior concentração da população menos favorecida nos setores censitários de exclusão social, com os piores indicadores, principalmente nas regiões norte, noroeste e nordeste da cidade e pequena área do extremo sudeste.

As classes menos favorecidas apresentam até 33,1\% dos responsáveis pelos domicílios sem renda nominal mensal e até $43,2 \%$ recebendo até dois salários mínimos mensalmente. A classe social com mais alta renda e maior nível de escolaridade localiza-se, predominantemente, na região centro-sul da malha urbana, onde os outros indicadores de habitação e de infraestrutura de saneamento básico apresentam as melhores condições ${ }^{30}$.

Neste estudo, a distribuição espacial dos coeficientes de incidência TB/HIV apresentou correspondência com níveis socioeconômicos dos moradores dos setores censitários, com as áreas com piores níveis apresentando maiores incidências, o que é coerente com a literatura, uma vez que muitos autores têm relacionado a ocorrência destas duas doenças a fatores como a pauperização das comunidades, a ruptura dos laços de interação social e a indisponibilidade de recursos para as comunidades do ponto de vista da saúde, habitação e políticas públicas ${ }^{12,15,17,27}$.

Ressalta-se que o agravamento das condições sociais e econômicas resulta em uma degradação significativa das condições de vida, aumentando a vulnerabilidade e, consequentemente, o risco de adoecer por TB/HIV ${ }^{6,7}$.

Os aspectos epidemiológicos da associação da TB com a AIDS representam grande desafio, considerando as dificuldades na organização das ações de controle das duas doenças, executadas por programas distintos, desarticulados e com políticas de controle em níveis diversos de atenção ${ }^{5,6}$. O controle da TB, associado ao nível de atenção primária, ainda enfrenta problemas relacionados ao desempenho deste nível de atenção, considerando a falta de vontade política em disponibilizar recursos e despreparo dos profissionais de saúde ${ }^{31,32}$.

Ao contrário, a assistência à AIDS, desde sua notificação no Brasil, por sua repercussão social, política e econômica, tem sido vislumbrada de forma mais efetiva, pois conta com recursos financeiros de organizações governamentais e não governamentais, nacionais e internacionais ${ }^{5,6}$. Além disso, o programa de controle da AIDS, no município se apresenta bem estruturado, com um Sistema de Vigilância eficiente, com programa de aconselhamento e testagem sorológica implantado em todos os serviços de atenção primária e especializados para o tratamento. Este fato contribui para que o município seja pólo de atração para o diagnóstico e tratamento da AIDS, o que, provavelmente incrementa a notificação de casos de doença, refletindo também na coinfecção ${ }^{33}$.

Embora todos os investimentos em uma política de atenção a AIDS, a infecção dupla (TB/HIV) representa desafio também duplo, diante da complexidade das duas doenças determinadas por um contexto biológico, social econômico e cultural, no âmbito individual e das coletividades, o que reforça a necessidade da colaboração e integração no nível local, entre programas nacionais da TB e HIV.Estudos, como este, permitem o reconhecimento de distintas realidades e podem contribuir para o equacionamento das várias dificuldades apontadas ${ }^{9}$.

Seus resultados permitem que o planejamento das ações, em qualquer nível de gestão e de forma rotineira, seja orientado para a 
priorização das regiões com maior carência social e consequentemente maiores incidências da doença ${ }^{27}$.

No caso de São José do Rio Preto, as análises realizadas através do LISA apontaram aglomerados de unidades espaciais significantes, os quais são uma boa indicação para priorização de áreas de maior risco. Seus resultados também contribuem para o estabelecimento de estratégias intersetoriais articuladas, com vistas à melhoria das condições de vida da população, em consonância com o SUS, implementando ações de prevenção e controle que considerem as limitações de acesso e percepção dos diferentes grupos populacionais que apresentam também diferentes graus de vulnerabilidade.

As limitações possíveis do estudo podem estar relacionadas ao fato de alguns doentes com TB não aceitarem submeterem-se aos testes para detecção do HIV. Outra limitação, como problemas no preenchimento das informações das fichas epidemiológicas, que resultou na não geocodificação de $12,5 \%$ dos casos notificados.

Este estudo possibilitou uma leitura adequada da distribuição geográfica espacial da co-infecção TB/HIV no município, que revelou, além das desigualdades intraurbanas, a localização das diferentes classes sociais, evidenciando os setores censitários que merecem maior atenção, considerando que as maiores incidências da co-infecção se concentram nas áreas com maior carência social.

Assim, aventamos a importância deste estudo como subsídio para um planejamento das políticas públicas inclusivas, orientado para as áreas com maior vulnerabilidade, com o propósito de intervir diretamente na realidade para minimizar as desigualdades socioespaciais existentes, fatores determinantes do processo saúdedoença, frequentemente banalizadas e negligenciadas.

\section{CONFLITO DE INTERESSE}

Os autores declaram não haver nenhum tipo de conflito de interesse no desenvolvimento do estudo.

\section{SUPORTE FINANCEIRO}

Projeto CNPq - processo - 410547/2006-9 - Multicêntrico - Edital MCT/CNPq/MS-SCTIE n. ${ }^{\circ}$ 25/2006 - Doenças Negligenciadas.

\section{REFERÊNCIAS}

1. Cock KM. HIV infection, tuberculosis and World AIDS Day, 2006. Int J Tuberc Lung Dis 2006; 10:1305.

2. BartlettJG. Tuberculosis and HIV Infection: Partners in Human Tragedy. In: Friedland G, Churchyard GJ, Nardell E, editors. Tuberculosis and HIV Coinfection: Current State of Knowledge and Research Priorities. Infect Dis J 2007; 196 (S124)

3. Organização Mundial de Saúde. Stop TB e HIV/SIDA. Quadro geral estratégico para reduzir o peso da TB/HIV. Geneva; 2002.

4. Range N, Ipuge Y, Obrien RJ, Egwaga SM, Mfinanga SG, Chonde TM, et al. Trend in HIV prevalence among tuberculosis patients in Tanzania, 1991-1998. Int J Tuberc Lung Dis 2001; 5:405-412.

5. Jamal LF, Moherdaui F. Tuberculose e infecção pelo HIV no Brasil: magnitude do problema e estratégias para o controle. Rev Saude Publica 2007; 41(supl 1): 104-110.

6. Vendramini SHF, Santos MLSG, Gazetta CE, Chiaravalloti-Neto F, Ruffino-Netto A Tuberculosis risk and sócio-economic level: a study of a city in the Brazilian south-east, 1998-2004. Int J Tuberc Lung Dis 2006; 10:1231-1235.

7. Santos MLSG, Vendramini SHF, Gazetta CE, Oliveira SAC, Villa TCS. Poverty: socioeconomic characterization at tuberculosis. Rev Lat Am Enfermagem 2007; 15:762-767.

8. Santos Filho ET. Política de TB no Brasil: Uma perspectiva da sociedade civil: Tempos de mudanças no controle da tuberculose no Brasil. Rio de Janeiro: Open Society Institute; 2006
9. Friedland G, Harries A, Coetzee D. Implementation Issues in Tuberculosis/HIV Program Collaboration and Integration: 3 Case Studies. In: Friedland G, Churchyard GJ, Nardell E. Tuberculosis and HIV Coinfection: Current State of Knowledge and Research Priorities. Infect Dis J 2007; 196:(S115).

10. Ministério da Saúde. Sistema de Informação em Saúde. DATASUS. Brasília; 2004 Disponível em: http://dtr2004.saude.gov.br/sinanweb/novo. Acessado em 17 de setembro de 2008.

11. Leal MC, Szwarcwald CL. Características da mortalidade neonatal no Estado do Rio de Janeiro na década de 80: uma visão espaço-temporal. Rev Saude Publica 1997; 31:457-465.

12. Skaba DA, Carvalho M, Sá BC, Martins PC, Terron SL. Geoprocessamento dos dados da saúde: o tratamento dos endereços. Cad Saude Publica 2004; 20:1753-1756.

13. Porter J. Geographical information systems (GIS) and the tuberculosis DOTS strategy. Int Trop Med Health 1999; 4:631-633.

14. Moonan P, Bayona B, Quitugua T, Oppong J, Dunbar D, Jost Jr K, et al. Using GIS technology to identify areas of tuberculosis transmission and incidence. Int $\mathrm{J}$ Health Geogr 2004; 3:23.

15. Rodrigues-Jr A, Netto-Ruffino A, Castilho EA. Distribuição espacial da coinfecção M. Tuberculosis/HIV no Estado de São Paulo, 1991-2001. Rev Saude Publica 2006; 40:265-270.

16. Souza WV, Ximenes R, Albuquerque MFM, Lapa TM, Portugal JL, Lima MLC, et al. The use of socioeconomic factors in mapping tuberculosis risk areas in a city of northeastern Brazil. Rev Panam Salud Publica 2000; 8:403-410.

17. Chan-Yeung M, Yeh AGO, Tam CM, Kam KM, Leung CC, Yew WW, et al. Sociodemographic and geographic indicators and distribution of tuberculosis in Hong Kong: a spatial analysis. Int J Tuberc Lung Dis 2005; 9:1320-1326.

18. Munch Z, Van Lill SWP, Booysen CN, Zietsman HL, Enarson DA, Beyers N Tuberculosis transmission patterns in a high-incidence area: a spatial analysis. Int J Tuberc Lung Dis 2003; 7:271-277.

19. Vieira RCA, Prado TN, Siqueira MG, Dietze R, Maciel ELN. Distribuição espacial dos casos novos de tuberculose em Vitória, Estado do Espírito Santo, no período entre 2000 e 2005. Rev Soc Bras Med Trop 2008; 41:82-86.

20. Cliff AD, Ord JK. Spatial Process: Models and Applications. London: Pion; 1981.

21. Andrade CLT, Szwarcwald CL. Análise espacial da mortalidade neonatal precoce no Município do Rio de Janeiro, 1995-1996. Rev Saude Publica 2001; 17:1199-1210.

22. Shapiro SS, Wilk MB. An analysis of variance test for normality (complete samples) Biometrika 1965; 52:591-611.

23. Anselin L. SpaceStat Tutorial. A workbook for using SpaceStat in the analysis of spatial data. Morgantown: Regional Institute, West Virginia University; 1992.

24. Silveira JM, Sassi RAM, Oliveira-Netto IC, Hetzel JL. Prevalência e fatores associado à tuberculose em pacientes soropositivos para o vírus da imunodeficiência humana em centro de referência para tratamento da síndrome da imunodeficiência adquirida na região sul do Rio Grande do Sul. J Bras Pneumol 2006; 32:48-55.

25. Instituto Brasileiro de Geografia e Estatística. IBGE [homepage na internet]. Brasília: Ministério do Planejamento, Orçamento e Gestão; [Acesso 18 julho 2008]. Disponível em: http://www.ibge.gov.br/home/estatistica/populacao/ censo2000/ ufs.php?tipo=31o/tabela13_1.shtm/.

26. Santos MLSG, Ponce MAS, Vendramini SHF, Villa TCS, Santos NSGM, Wysocki AD et.al. A dimensão epidemiológica da coinfecção TB/HIV. Rev Lat Am Enfermagem 2009; 17:683-688.

27. Davidow AL, Mangura BT, Napolitano EC, Reichman LB. Rethinking the socioeconomics and geography of tuberculosis among foriengn-born residents of New Jersey, 1994-1999. Am Public Health J 2003; 93:1007-1012.

28. Fonseca MG, Bastos FI, Derriço M, Andrade CLT, Travassos C, Szwarcwald CL. AIDS e grau de escolaridade no Brasil: evolução temporal de 1986 a 1996. Cad Saude Publica 2000; 16(supl 1):77-87.

29. Federação das Indústrias do Estado do Rio de Janeiro (FIRJAN). Índice FIRJAN de Desenvolvimento Municipal. Rio de Janeiro; 2008. Disponível em: http://ifdm.firjan. org.br/media/2005/Publicacao_IFDM2.pdf.

30. Vieira $A B$. Mapeamento da exclusão social em cidades médias: interfaces da Geografia Econômica com a Geografia Política [Tese de doutorado]. [Presidente Prudente]: Universidade Estadual Paulista; 2009.

31. Ruffino-Netto A, Villa TC. Tuberculosis treatment: DOTS implementation in some regions of Brazil background and regional features. Ribeirão Preto: Millennium Institute - REDE TB, Brazilian Tuberculosis Research Network; 2008.

32. Monroe AA, Gonzalles RIC, Palha PF, Sassaki CM, Ruffino-Netto A, Vendramini SHF et al. Envolvimento de equipes da atenção básica à saúde no controle da tuberculose Rev Esc Enferm USP 2008; 42:262-267.

33. Vendramini SHF, Gazetta CE, Cury MRCO, Villa TCS, Ruffino-Netto A. DOTS Implementation in São José do Rio Preto. In: Ruffino-Netto A, Villa TC, editors. Tuberculosis treatment: DOTS implementation in some regions of Brazil background and regional features. Ribeirão Preto: Millennium Institute, REDE TB, Brazilian Tuberculosis Research Network; 2008. p.118-126. 